\title{
Singular and Degenerate Boundary Value Problems Related to the Electricity Theory
}

\author{
Maria-Magdalena Boureanu' ${ }^{1}$ and Andaluzia Matei $^{2}$ \\ ${ }^{1}$ Department of Applied Mathematics, University of Craiova, 200585 Craiova, Romania \\ ${ }^{2}$ Department of Mathematics, University of Craiova, 200585 Craiova, Romania \\ Correspondence should be addressed to Andaluzia Matei; andaluziamatei2000@yahoo.com
}

Received 9 December 2014; Accepted 15 February 2015

Academic Editor: Zhen-Lai Han

Copyright (c) 2015 M.-M. Boureanu and A. Matei. This is an open access article distributed under the Creative Commons Attribution License, which permits unrestricted use, distribution, and reproduction in any medium, provided the original work is properly cited.

\begin{abstract}
The present paper draws attention to the weak solvability of a class of singular and degenerate problems with nonlinear boundary conditions. These problems derive from the electricity theory serving as mathematical models for physical phenomena related to the anisotropic media with "perfect" insulators or "perfect" conductors points. By introducing an appropriate weighted Sobolev space to the mathematical literature, we establish an existence and uniqueness result.
\end{abstract}

\section{Introduction}

During the last decades there has been a high interest in the study of the following class of problems:

$$
\begin{gathered}
\operatorname{div}(\mu(\mathbf{x}) \nabla u(\mathbf{x}))+f(\mathbf{x})=0 \quad \text { in } \Omega, \\
u(\mathbf{x})=0 \quad \text { on } \partial \Omega,
\end{gathered}
$$

where $\Omega$ is an arbitrary domain, $\mu$ is a nonnegative weight, and $f$ is a nonlinear function satisfying certain conditions. If the operator involved in (1) is a uniformly elliptic operator, then the existence of solutions can be ensured by classical critical point theory means; see, for example, $[1,2]$. But when this is not the case and $\mu$ is a function that has zeros at some points or it is unbounded, the problem becomes much more mathematically complicated and thus the mathematical interest increases. However, the interest in differential operators with degeneracies or singularities is not purely mathematical, since they are used in the study of several physical phenomena. Indeed, the restrictions of the type $\mu(x) \geq$ const. $>0$ for all $x$, or $\mu(x) \leq$ const. $<\infty$ for all $x$, are not natural; thus it is worth struggling with all the difficulties that appear in the calculus otherwise. As for the methods utilized to overcome these inconveniences, they depend on the problem. Many papers discuss the existence of solutions for (1) when the weight has the particular form $\mu(x)=|x|^{b}$, so that, under some appropriate hypotheses on $b$, a Caffarelli-Kohn-Nirenberg inequality can be applied; see, for example, [3-5] and the references therein.

The present paper approaches a different situation by preserving a higher degree of generality on the weight. Let $\Omega \subset \mathbb{R}^{2}$ be an open, bounded, connected subset, with Lipschitz continuous boundary $\Gamma$ partitioned in three measurable parts $\Gamma_{1}, \Gamma_{2}, \Gamma_{3}$ such that the Lebesgue measure of $\Gamma_{1}$ is positive. We are interested in the following problem.

Problem 1. Find $u: \bar{\Omega} \rightarrow \mathbb{R}$ such that

$$
\begin{gathered}
\operatorname{div}(\beta(\mathbf{x}) \nabla u(\mathbf{x}))+q_{0}(\mathbf{x})=0 \quad \text { in } \Omega, \\
u(\mathbf{x})=0 \quad \text { on } \Gamma_{1}, \\
\beta(\mathbf{x}) \frac{\partial u}{\partial \nu}(\mathbf{x})=q_{2}(\mathbf{x}) \quad \text { on } \Gamma_{2}, \\
\beta(\mathbf{x}) \frac{\partial u}{\partial \nu}(\mathbf{x})=-k \frac{u(\mathbf{x})}{\sqrt{u^{2}(\mathbf{x})+\alpha}} \text { on } \Gamma_{3},
\end{gathered}
$$

where $\boldsymbol{\nu}$ denotes, as usual, the outward unit normal to $\Gamma, \beta$ satisfies $\beta, \beta^{-1} \in L^{2}(\Omega)$ and $\beta>0$ a.e. in $\Omega$ with $\inf _{\bar{\Omega}} \beta=0$, 
$\sup _{\bar{\Omega}} \beta=\infty$, and the other functions satisfy $k, \alpha>0, q_{0} \in$ $L^{2}(\Omega)$, and $q_{2} \in L^{\infty}\left(\Gamma_{2}\right)$.

Working under the previous conditions, we want to prove that Problem 1 admits a unique weak solution. Our way of surpassing the difficulties created by the presence of the possibly singular or degenerate points is by conducting our study in a weighted Sobolev-type space that will be introduced in the next section. This space appears for the first time in the mathematical literature, although it is similar to the weighted Sobolev space used in [6]. These two studies share the same kind of space to work on because of the following common aspect: they both treat degenerate problems and in both cases we only require $u=0$ on a nonempty subset of the boundary instead of the usual zero Dirichlet boundary condition imposed on the entire boundary. This fact can be correlated with concrete applications and represents a novel trait of our research. Despite some similarities, there are some clear differences between problem (2)-(5) and the problem treated in [6]: the problem from here admits singular points, and, more importantly, it is addressed to a different range of applications. To be specific, while the problem from [6] is a frictional contact problem that arrives from the theory of elasticity, boundary value problems like (2)-(5) arrive from the theory of electricity. Also, we mention that equations of type (2) were introduced as models for various physical phenomena connected to the equilibrium of anisotropic media that possibly are somewhere "perfect" insulators or "perfect" conductors; see [7, page 79].

We will discuss Problem 1 in Section 3. But before that, we present the abstract framework and some preliminary results that serve our goal.

\section{Functional Setting and Preliminaries}

Let $N \geq 2$ and $\Omega \subset \mathbb{R}^{N}$ be a bounded domain with Lipschitz continuous boundary $\Gamma$. Let $\zeta: \Omega \rightarrow \mathbb{R}$ be a function verifying the hypotheses

$$
\begin{gathered}
\zeta \in L^{4}(\Omega), \quad \zeta^{-1} \in L^{4}(\Omega), \quad \zeta(\mathbf{x}) \neq 0 \text { a.e. on } \Omega, \\
\inf _{\mathbf{x} \in \bar{\Omega}} \zeta^{2}(\mathbf{x})=0, \quad \sup _{\mathbf{x} \in \bar{\Omega}} \zeta^{2}(\mathbf{x})=\infty .
\end{gathered}
$$

As an example of such function, one can take $\zeta:(-1,1) \times$ $(-1,1) \rightarrow \mathbb{R}$ such that

$$
\zeta\left(x_{1}, x_{2}\right)= \begin{cases}1 & \text { for } x_{1} \leq 0 \\ \left(\frac{1-x_{1}}{x_{2}}\right)^{1 / 4} & \text { for } x_{1}>0, x_{2}>0 \\ \left(1-x_{1}\right)^{1 / 4} & \text { for } x_{1}>0, x_{2} \leq 0\end{cases}
$$

see also [8, Example 2.7].

Starting from the weight $\zeta$ described by assumptions (6) we can introduce a weighted Sobolev-type space

$$
H_{\zeta}^{1}(\Omega)=\left\{v: \Omega \longrightarrow \mathbb{R} \mid v \in L^{2}(\Omega), \zeta \nabla v \in L^{2}(\Omega)^{2}\right\}
$$

endowed with the inner product

$$
(u, v)_{H_{\zeta}^{1}(\Omega)}=(u, v)_{L^{2}(\Omega)}+(\zeta \nabla u, \zeta \nabla v)_{L^{2}(\Omega)^{2}}
$$

and the corresponding norm

$$
\|v\|_{H_{\zeta}^{1}(\Omega)}=\left(\|v\|_{L^{2}(\Omega)}^{2}+\|\zeta \nabla v\|_{L^{2}(\Omega)^{2}}^{2}\right)^{1 / 2} .
$$

Following [9], this weighted Sobolev space associated with norm (10) is a Hilbert space. Using similar arguments to those from [6, Lemma 3.1], one can deduce that if $v \in H_{\zeta}^{1}(\Omega)$, then $v \in W^{1,1}(\Omega)$, and, in addition, there exists $c_{E}=c_{E}(\Omega, \zeta)>0$ such that

$$
\|v\|_{W^{1,1}(\Omega)} \leq c_{E}\|v\|_{H_{\zeta}^{1}(\Omega)}, \quad \forall v \in H_{\zeta}^{1}(\Omega)
$$

On the other hand, according to the Gagliardo trace theorem (see [10]), there exists a linear continuous operator $\gamma$ : $W^{1,1}(\Omega) \rightarrow L^{1}(\Gamma)$, called the Gagliardo trace operator, such that

$$
\gamma v=\left.v\right|_{\Gamma} \text { if } v \in W^{1,1}(\Omega) \cap C(\bar{\Omega})
$$

and there exists $c_{G}=c_{G}(\Omega)>0$ such that

$$
\|\gamma v\|_{L^{1}(\Gamma)} \leq c_{G}\|v\|_{W^{1,1}(\Omega)} \quad \text { for each } v \in W^{1,1}(\Omega) .
$$

Everywhere below, by $\gamma$ we will denote the Gagliardo trace operator. Taking into consideration the above said,

$$
\text { for every } v \in H_{\zeta}^{1}(\Omega) \text { we deduce that } \gamma v \in L^{1}(\Gamma) \text {. }
$$

In order to search for weak solutions for problem (2)-(5), we will work on the following subspace of $H_{\zeta}^{1}(\Omega)$ :

$$
V_{\zeta}=\left\{v \in H_{\zeta}^{1}(\Omega) \mid \gamma v=0 \text { a.e. on } \Gamma_{1}\right\} \text {, }
$$

where $\Gamma_{1} \subset \Gamma$ with meas $\left(\Gamma_{1}\right)>0$. As a closed subspace of the Hilbert space $\left(H_{\zeta}^{1}(\Omega),(\cdot, \cdot)_{H_{\zeta}^{1}(\Omega)},\|\cdot\|_{H_{\zeta}^{1}(\Omega)}\right)$, the space $\left(V_{\zeta},(\cdot, \cdot)_{H_{\zeta}^{1}(\Omega)},\|\cdot\|_{H_{\zeta}^{1}(\Omega)}\right)$ is also a Hilbert space. Besides,

$$
(u, v)_{V_{\zeta}}=\int_{\Omega} \zeta^{2}(\mathbf{x}) \nabla u(\mathbf{x}) \cdot \nabla v(\mathbf{x}) d x
$$

defines an inner product on the space $V_{\zeta}$. Let us denote by $\|\cdot\|_{V_{\zeta}}$ the norm induced by the inner product (16),

$$
\|v\|_{V_{\zeta}}=\|\zeta \nabla v\|_{L^{2}(\Omega)^{2}} \quad \forall v \in V_{\zeta} .
$$

By adapting the proof of [6, Lemma 3.2] to our present situation, one can see that the norms $\|\cdot\|_{H_{\zeta}^{1}(\Omega)}$ and $\|\cdot\|_{V_{\zeta}}$ are equivalent on $V_{\zeta}$. Consequently, $\left(V_{\zeta},(\cdot, \cdot)_{V_{\zeta}},\|\cdot\|_{V_{\zeta}}\right)$ is a Hilbert space too.

Let us proceed by recalling the following tool.

Theorem 2. Suppose $\left(X,(\cdot, \cdot)_{X},\|\cdot\|_{X}\right)$ is a Hilbert space. Let A : $X \rightarrow X$ be a Lipschitz continuous operator with the property that

there exists $m>0$ such that

$$
(A u-A v, u-v)_{X} \geq m\|u-v\|_{X}^{2} \quad \forall u, v \in X .
$$


Let $j: X \rightarrow \overline{\mathbb{R}}$ be a proper, convex, and continuous functional. Then, given $f \in X$, there exists a unique element $u \in X$ such that

$$
(A u, v-u)_{X}+j(v)-j(u) \geq(f, v-u)_{X} \quad \forall v \in X .
$$

Moreover, the solution depends Lipschitz continuously on $f$.

For a proof of the above theorem, the reader can consult, for example, [11]. We end this section with three preliminary results on convex analysis.

Theorem 3 (see [12, Theorem 6.2.1.]). Let $X$ be a reflexive Banach space, and let $f: X \rightarrow \mathbb{R}$ be Gâteaux differentiable. Then the following conditions are equivalent:

(i) $f$ is convex;

(ii) one has

$$
f(u)-f(v) \geq\left(f^{\prime}(v), u-v\right)_{X^{\star} \times X} \quad \forall u, v \in X,
$$

where $X^{\star}$ denotes the dual of the space $X$.

Theorem 4 (see [11, Proposition 1.59]). Let X be a real normed space and let $f: X \rightarrow \overline{\mathbb{R}}$ be a proper convex lower semicontinuous functional. Then $\partial f(u) \neq \emptyset$ for all $u \in \operatorname{int}(\operatorname{dom}(f))$; that is, $f$ is subdifferentiable on $\operatorname{int}(\operatorname{dom}(f))$.

Theorem 5 (see [11, Proposition 1.60]). Let $X$ be a real normed space and let $f: X \rightarrow \overline{\mathbb{R}}$ be convex. Assume $u \in X$ and $f(u) \in \mathbb{R}$. If $f^{\prime}(u)$ exists as a Gâteaux derivative, then $\partial f(u)=$ $\left\{f^{\prime}(u)\right\}$.

Now we are ready to present our existence and uniqueness result.

\section{Main Result}

In what follows, we consider $\zeta=\sqrt{\beta}$, where $\beta$ is the function from Problem 1. In order to deliver a weak formulation of Problem 1 , we assume that $u$ is a strong solution. We multiply (2) by a function $v \in C^{\infty}(\bar{\Omega})$, and, after integration by parts, we obtain

$$
\begin{aligned}
\int_{\Omega} \beta(\mathbf{x}) \nabla u(\mathbf{x}) \cdot \nabla v(\mathbf{x}) d x= & \int_{\Omega} q_{0}(\mathbf{x}) v(\mathbf{x}) d x \\
& +\int_{\Gamma} \beta(\mathbf{x}) \frac{\partial u}{\partial \nu}(\mathbf{x}) v(\mathbf{x}) d \Gamma .
\end{aligned}
$$

Due to boundary conditions (3)-(5), we infer that

$$
\begin{aligned}
\int_{\Omega} \beta(\mathbf{x}) \nabla u(\mathbf{x}) \cdot \nabla v(\mathbf{x}) d x \\
=\int_{\Omega} q_{0}(\mathbf{x}) v(\mathbf{x}) d x+\int_{\Gamma_{1}} \beta(\mathbf{x}) \frac{\partial u}{\partial v}(\mathbf{x}) v(\mathbf{x}) d \Gamma \\
\quad+\int_{\Gamma_{2}} q_{2}(\mathbf{x}) v(\mathbf{x}) d \Gamma \\
\quad-k \int_{\Gamma_{3}} \frac{u(\mathbf{x}) v(\mathbf{x})}{\sqrt{u^{2}(\mathbf{x})+\alpha}} d \Gamma \quad \forall v \in C^{\infty}(\bar{\Omega}) .
\end{aligned}
$$

The density result $\overline{C^{\infty}(\bar{\Omega})}=W^{1,1}(\Omega)$ implies that

$$
\begin{aligned}
& \int_{\Omega} \beta(\mathbf{x}) \nabla u(\mathbf{x}) \cdot \nabla v(\mathbf{x}) d x \\
& =\int_{\Omega} q_{0}(\mathbf{x}) v(\mathbf{x}) d x+\int_{\Gamma_{1}} \beta(\mathbf{x}) \frac{\partial u}{\partial v}(\mathbf{x}) \gamma v(\mathbf{x}) d \Gamma \\
& \quad+\int_{\Gamma_{2}} q_{2}(\mathbf{x}) \gamma v(\mathbf{x}) d \Gamma \\
& \quad-k \int_{\Gamma_{3}} \frac{\gamma u(\mathbf{x}) \gamma v(\mathbf{x})}{\sqrt{(\gamma u)^{2}(\mathbf{x})+\alpha}} d \Gamma \quad \forall v \in W^{1,1}(\Omega) .
\end{aligned}
$$

This allows us to give the definition of the weak solution to our problem.

Definition 6. By a weak solution for Problem 1 one understands a function $u \in V_{\zeta}$ which verifies

$$
\begin{aligned}
& \int_{\Omega} \beta(\mathbf{x}) \nabla u(\mathbf{x}) \cdot \nabla v(\mathbf{x}) d x \\
& =\int_{\Omega} q_{0}(\mathbf{x}) v(\mathbf{x}) d x+\int_{\Gamma_{2}} q_{2}(\mathbf{x}) \gamma v(\mathbf{x}) d \Gamma \\
& \quad-k \int_{\Gamma_{3}} \frac{\gamma u(\mathbf{x}) \gamma v(\mathbf{x})}{\sqrt{(\gamma u)^{2}(\mathbf{x})+\alpha}} d \Gamma,
\end{aligned}
$$

for all $v \in V_{\zeta}$.

Let us introduce now the main result of our paper.

Theorem 7. There exists a unique weak solution to Problem 1.

To provide a proof for this theorem we rely on Theorem 2, so we prove several auxiliary lemmas.

Lemma 8. There exists $A: V_{\zeta} \rightarrow V_{\zeta}$ such that

$$
(A u-A v, u-v)_{V_{\zeta}}=\|u-v\|_{V_{\zeta}}^{2} \quad \forall u, v \in V_{\zeta} .
$$

Proof. We introduce the form

$$
a: V_{\zeta} \times V_{\zeta} \longrightarrow \mathbb{R}, \quad a(u, v)=\int_{\Omega} \beta(\mathbf{x}) \nabla u(\mathbf{x}) \cdot \nabla v(\mathbf{x}) d x
$$

and we claim that $a$ is well defined, bilinear, and continuous. Indeed, since $u, v \in H_{\zeta}^{1}(\Omega)$ and $\beta=\zeta^{2}$, function $a$ is well defined. Also, from the linearity of the gradient and of the integral operator, one can immediately see that $a$ is bilinear. Then, to show the continuity of $a$, it is enough to notice that, by the Hölder inequality, we arrive at

$$
|a(u, v)| \leq\|\zeta \nabla u\|_{L^{2}(\Omega)}\|\zeta \nabla v\|_{L^{2}(\Omega)}
$$

that is,

$$
|a(u, v)| \leq\|u\|_{V_{\zeta}}\|v\|_{V_{\zeta}} \text {. }
$$


With that, our claim is proved and we can apply Riesz's representation theorem to deduce that there exists

$$
A: V_{\zeta} \longrightarrow V_{\zeta}, \quad\left(A v_{1}, v_{2}\right)_{V_{\zeta}}=a\left(v_{1}, v_{2}\right) .
$$

We have

$$
\begin{aligned}
& (A u-A v, u-v)_{V_{\zeta}} \\
& =\int_{\Omega} \beta(\mathbf{x})\|\nabla u(\mathbf{x})-\nabla v(\mathbf{x})\|^{2} d x \\
& =\|u-v\|_{V_{\zeta}}^{2} \quad \forall u, v \in V_{\zeta},
\end{aligned}
$$

where, as usual, $\|\cdot\|$ denotes the Euclidean norm on $\mathbb{R}^{2}$. Hence our proof is finished.

Let us define now the following application:

$$
\begin{gathered}
j: V_{\zeta} \longrightarrow \mathbb{R}, \\
j(u)=\int_{\Gamma_{3}} k\left[\sqrt{(\gamma u)^{2}(\mathbf{x})+\alpha^{2}}-\alpha\right] d \Gamma .
\end{gathered}
$$

Lemma 9. The functional $j$ is well defined, continuous, Gâteaux differentiable, and convex.

Proof. Note that

$$
\begin{aligned}
& \left|\sqrt{(\gamma u)^{2}(\mathbf{x})+\alpha^{2}}-\alpha\right| \\
& =\frac{|\gamma u(\mathbf{x})|}{\sqrt{(\gamma u)^{2}(\mathbf{x})+\alpha^{2}}+\alpha}|\gamma u(\mathbf{x})| \leq|\gamma u(\mathbf{x})| .
\end{aligned}
$$

Since $u \in V_{\zeta}$, we have that $\gamma u \in L^{1}(\Gamma)$; thus

$$
\int_{\Gamma_{3}}\left|\sqrt{(\gamma u)^{2}(\mathbf{x})+\alpha^{2}}-\alpha\right| d \Gamma<\infty,
$$

and consequently $j$ is well defined. Also, it is not difficult to see that $j$ is continuous.

In what concerns the Gâteaux differentiability of $j$, for all $u, v \in V_{\zeta}$ we have

$$
\begin{aligned}
\lim _{t \rightarrow 0^{+}} & \frac{j(u+t v)-j(u)}{t} \\
\quad & \left.\frac{d}{d t} j(u+t v)\right|_{t=0} \\
& =\left.k \frac{d}{d t} \int_{\Gamma_{3}}\left[\sqrt{(\gamma(u+t v))^{2}(\mathbf{x})+\alpha^{2}}-\alpha\right] d \Gamma\right|_{t=0} .
\end{aligned}
$$

By Lebesgue's Theorem, the differentiation under the integral is allowed if, for $t$ close to zero, the term $\sqrt{(\gamma(u+t v))^{2}(\mathbf{x})+\alpha^{2}}-\alpha$ is dominated by a fixed $L^{1}$ function which does not depend on $t$. Indeed, by (32),

$$
\begin{aligned}
\left|\sqrt{(\gamma(u+t v))^{2}(\mathbf{x})+\alpha^{2}}-\alpha\right| & \leq|\gamma(u+t v)(\mathbf{x})| \\
& \leq|\gamma u(\mathbf{x})|+|\gamma v(\mathbf{x})|,
\end{aligned}
$$

for $|t|<1$. Therefore,

$$
\begin{aligned}
\lim _{t \rightarrow 0^{+}} & \frac{j(u+t v)-j(u)}{t} \\
\quad & \left.k \int_{\Gamma_{3}} \frac{\partial}{\partial t}\left[\sqrt{[\gamma u(\mathbf{x})+t \gamma v(\mathbf{x})]^{2}+\alpha^{2}}-\alpha\right]\right|_{t=0} d \Gamma \\
& =k \int_{\Gamma_{3}} \frac{\gamma u(\mathbf{x}) \gamma v(\mathbf{x})}{\sqrt{(\gamma u)^{2}(\mathbf{x})+\alpha^{2}}} d \Gamma .
\end{aligned}
$$

It is clear that the application which maps $v \in V_{\zeta}$ into $k \int_{\Gamma_{3}}\left(\gamma u(\mathbf{x}) \gamma v(\mathbf{x}) / \sqrt{(\gamma u)^{2}(\mathbf{x})+\alpha^{2}}\right) d \Gamma$ is linear, so we prove its continuity. We have

$$
\left|k \int_{\Gamma_{3}} \frac{\gamma u(\mathbf{x}) \gamma v(\mathbf{x})}{\sqrt{(\gamma u)^{2}(\mathbf{x})+\alpha^{2}}} d \Gamma\right| \leq k \int_{\Gamma_{3}}|\gamma v(\mathbf{x})| d \Gamma .
$$

Using (13), (11), and the equivalence of norms $\|\cdot\|_{V_{\zeta}}$ and $\| \cdot$ $\|_{H_{\zeta}^{1}(\Omega)}$ on $V_{\zeta}$, there exists $k_{0}$ such that

$$
\left|k \int_{\Gamma_{3}} \frac{\gamma u(\mathbf{x}) \gamma v(\mathbf{x})}{\sqrt{(\gamma u)^{2}(\mathbf{x})+\alpha^{2}}} d \Gamma\right| \leq k_{0}\|v\|_{V_{\zeta}}
$$

for all $v \in V_{\zeta}$. This way, we have obtained that $j$ is Gâteaux differentiable and

$$
\left(j^{\prime}(u), v\right)_{V_{\zeta}}=k \int_{\Gamma_{3}} \frac{\gamma u(\mathbf{x}) \gamma v(\mathbf{x})}{\sqrt{(\gamma u)^{2}(\mathbf{x})+\alpha^{2}}} d \Gamma .
$$

As for convexity, due to Theorem 3, it is enough to show that

$$
j(u)-j(v) \geq\left(j^{\prime}(v), u-v\right)_{V_{\zeta}} \quad \forall u, v \in V_{\zeta}
$$

that is,

$$
\begin{aligned}
& \int_{\Gamma_{3}} {\left[\sqrt{(\gamma \nu)^{2}(\mathbf{x})+\alpha^{2}}-\alpha\right] d \Gamma } \\
&-\int_{\Gamma_{3}}\left[\sqrt{(\gamma u)^{2}(\mathbf{x})+\alpha^{2}}-\alpha\right] d \Gamma \\
& \geq \int_{\Gamma_{3}} \frac{\gamma u(\mathbf{x})[\gamma v(\mathbf{x})-\gamma u(\mathbf{x})]}{\sqrt{(\gamma u)^{2}(\mathbf{x})+\alpha}} d \Gamma
\end{aligned}
$$

for all $u, v \in V_{\zeta}$. To do so, we start from the fact that

$$
\begin{gathered}
\sqrt{\left[(\gamma v)^{2}(\mathbf{x})+\alpha^{2}\right]\left[(\gamma u)^{2}(\mathbf{x})+\alpha^{2}\right]} \\
\geq|\gamma u(\mathbf{x})||\gamma v(\mathbf{x})|+\alpha^{2},
\end{gathered}
$$

for all $u, v \in V_{\zeta}$. This immediately leads to

$$
\sqrt{\left[(\gamma v)^{2}(\mathbf{x})+\alpha^{2}\right]\left[(\gamma u)^{2}(\mathbf{x})+\alpha^{2}\right]}
$$

$$
-\alpha^{2}-(\gamma u)^{2}(\mathbf{x}) \geq \gamma u(\mathbf{x}) \gamma v(\mathbf{x})-(\gamma u)^{2}(\mathbf{x}) ;
$$


hence

$$
\begin{gathered}
\sqrt{(\gamma v)^{2}(\mathbf{x})+\alpha^{2}}-\sqrt{(\gamma u)^{2}(\mathbf{x})+\alpha^{2}} \\
\geq \frac{\gamma u(\mathbf{x})[\gamma v(\mathbf{x})-\gamma u(\mathbf{x})]}{\sqrt{(\gamma u)^{2}(\mathbf{x})+\alpha^{2}}}
\end{gathered}
$$

and (40) holds true. With this, we conclude the proof of the lemma.

Furthermore, the following result takes place.

Lemma 10. There exists a unique element $f \in V_{\zeta}$ such that

$$
\begin{gathered}
\int_{\Omega} q_{0}(\mathbf{x}) v(\mathbf{x}) d x+\int_{\Gamma_{2}} q_{2}(\mathbf{x}) \gamma v(\mathbf{x}) d \Gamma \\
=(f, v)_{V_{\zeta}} \quad \forall v \in V_{\zeta} .
\end{gathered}
$$

Proof. We introduce

$$
\begin{gathered}
\Phi: V_{\zeta} \longrightarrow \mathbb{R} \\
\Phi(v)=\int_{\Omega} q_{0}(\mathbf{x}) v(\mathbf{x}) d x+\int_{\Gamma_{2}} q_{2}(\mathbf{x}) \gamma v(\mathbf{x}) d \Gamma
\end{gathered}
$$

and we claim that $\Phi$ is well defined, linear, and continuous. Indeed, the form $\Phi$ is well defined because $q_{0}, v \in L^{2}(\Omega)$, $q_{2} \in L^{\infty}\left(\Gamma_{2}\right)$, and $\gamma v \in L^{1}(\Gamma)$. The linearity of $\Phi$ follows easily from the linearity of the integral operator. Let us focus on the continuity of $\Phi$. First, by the Hölder inequality,

$$
\left|\int_{\Omega} q_{0}(\mathbf{x}) v(\mathbf{x}) d x\right| \leq\left\|q_{0}\right\|_{L^{2}(\Omega)}\|v\|_{L^{2}(\Omega)}
$$

for all $v \in V_{\zeta}$. Then, by relation (10), we find that

$$
\left|\int_{\Omega} q_{0}(\mathbf{x}) v(\mathbf{x}) d x\right| \leq\left\|q_{0}\right\|_{L^{2}(\Omega)}\|v\|_{H_{\zeta}^{1}(\Omega)}
$$

for all $v \in V_{\zeta}$. At the same time,

$$
\left|\int_{\Gamma_{2}} q_{2}(\mathbf{x}) \gamma v(\mathbf{x}) d \Gamma\right| \leq\left\|q_{2}\right\|_{L^{\infty}\left(\Gamma_{2}\right)}\|\gamma v\|_{L^{1}(\Gamma)} .
$$

By (13) and (11) it follows that

$$
\left|\int_{\Gamma_{2}} q_{2}(\mathbf{x}) \gamma v(\mathbf{x}) d \Gamma\right| \leq c_{G} c_{E}\left\|q_{2}\right\|_{L^{\infty}\left(\Gamma_{2}\right)}\|v\|_{H_{\zeta}^{1}(\Omega)}
$$

for all $v \in V_{\zeta}$. We take into account the equivalence of the norms $\|\cdot\|_{V_{\zeta}}$ and $\|\cdot\|_{H_{\zeta}^{1}(\Omega)}$ and the linearity of $\Phi$, and then we put together (46), (48), and (50) to deduce that $\Phi$ is continuous.

At this point, we have all the ingredients to apply Riesz's representation theorem. So, we deduce that there exists a unique element $f \in V_{\zeta}$ such that

$$
\Phi(v)=(f, v)_{V_{\zeta}} \quad \forall v \in V_{\zeta}
$$

and the proof is complete.
Keeping in mind all the above assumptions, notations, and results, we are able to introduce the following weak formulation.

Problem 11. Find $u \in V_{\zeta}$ such that

$$
\begin{gathered}
(A u, v-u)_{V_{\zeta}}+j(v)-j(u) \\
\geq(f, v-u)_{V_{\zeta}} \quad \forall v \in V_{\zeta},
\end{gathered}
$$

where $A, j$, and $f$ are the ones from (29), (31), and (45), respectively.

Lemma 12. A function $u \in V_{\zeta}$ is a weak solution to Problem 1 if and only if it is a solution to Problem 11.

Proof. Let $u \in V_{\zeta}$ be a weak solution to Problem 1, meaning that $u$ verifies Definition 6. Consequently,

$$
\begin{aligned}
& \int_{\Omega} \beta(\mathbf{x}) \nabla u(\mathbf{x}) \cdot[\nabla u(\mathbf{x})-\nabla v(\mathbf{x})] d x \\
& =\int_{\Omega} q_{0}(\mathbf{x})[u(\mathbf{x})-v(\mathbf{x})] d x \\
& \quad+\int_{\Gamma_{2}} q_{2}(\mathbf{x})[\gamma u(\mathbf{x})-\gamma v(\mathbf{x})] d \Gamma \\
& \quad-k \int_{\Gamma_{3}} \frac{\gamma u(\mathbf{x})[\gamma u(\mathbf{x})-\gamma v(\mathbf{x})]}{\sqrt{(\gamma u)^{2}(\mathbf{x})+\alpha}} d \Gamma \quad \forall v \in V_{\zeta} .
\end{aligned}
$$

Keeping in mind relations (26), (29), (39), and (45), by relation (40) it is obvious that $u$ is a solution to Problem 11.

Conversely, let us consider now $u \in V_{\zeta}$ to be a solution to Problem 11. Then $f-A u \in \partial j(u)$. Moreover, due to Lemma 9, we can apply Theorems 4 and 5 to infer that $f-A u=j^{\prime}(u)$. This yields

$$
(f, v)_{V_{\zeta}}-(A u, v)_{V_{\zeta}}=\left(j^{\prime}(u), v\right)_{V_{\zeta}} \quad \forall v \in V_{\zeta} .
$$

Taking into consideration (26), (29), (39), and (45), we obtain that $u$ is a weak solution to Problem 1 .

Finally, we can conclude our main result.

Proof of Theorem 7. Based on the previous results, the proof of Theorem 7 is immediate. Indeed, by Lemma 12, it is enough to prove that Problem 11 has a unique solution. To this end, we use Lemmas 8 and 9 and we apply Theorem 2 .

\section{Conflict of Interests}

The authors declare that there is no conflict of interests regarding the publication of this paper.

\section{References}

[1] A. Ambrosetti and P. H. Rabinowitz, "Dual variational methods in critical point theory and applications," Journal of Functional Analysis, vol. 7, pp. 349-381, 1973. 
[2] P. H. Rabinowitz, "Variational methods for nonlinear elliptic eigenvalue problems," Indiana University Mathematics Journal, vol. 23, pp. 729-754, 1974.

[3] M. Ghergu and V. Rădulescu, "Singular elliptic problems with lack of compactness," Annali di Matematica Pura ed Applicata, vol. 185, no. 1, pp. 63-79, 2006.

[4] G. M. Bisci and V. Rădulescu, "Bifurcation analysis of a singular elliptic problem modelling the equilibrium of anisotropic continuous media," Topological Methods in Nonlinear Analysis. In press.

[5] M. Yang and Z. Shen, "The effect of domain topology on the number of positive solutions for singular elliptic problems involving the Caffarelli-Kohn-Nirenberg inequalities," Journal of Mathematical Analysis and Applications, vol. 334, no. 1, pp. 273-288, 2007.

[6] M. Boureanu and A. Matei, "Weak solutions for antiplane models involving elastic materials with degeneracies," Zeitschrift für Angewandte Mathematik und Physik, vol. 61, no. 1, pp. 73-85, 2010.

[7] R. Dautray and J.-L. Lions, Mathematical Analysis and Numerical Methods for Science and Technology, Volume 1: Physical Origins and Classical Methods, Springer, Berlin, Germany, 1985.

[8] S. Leonardi, "The best constant in weighted Poincaré and Friedrichs inequalities," Rendiconti del Seminario Matematico della Università di Padova, vol. 92, pp. 195-208, 1994.

[9] S. Leonardi, "Solvability of degenerate quasilinear elliptic equations," Nonlinear Analysis: Theory, Methods \& Applications, vol. 26, no. 6, pp. 1053-1060, 1996.

[10] E. Gagliardo, "Caratterizzazioni delle tracce sulla frontiera relative ad alcune classi di funzioni in $n$ variabili," Rendiconti del Seminario Matematico della Università di Padova, vol. 27, pp. 284-305, 1957.

[11] W. Han and M. Sofonea, Quasistatic Contact Problems in Viscoelasticity and Viscoplasticity, Studies in Advanced Mathematics 30, American Mathematical Society, Providence/ International Press, Somerville, Mass, USA, 2002.

[12] A. J. Kurdila and M. Zabarankin, Convex Functional Analysis, Birkhäuser, Basel, Switzerland, 2005. 


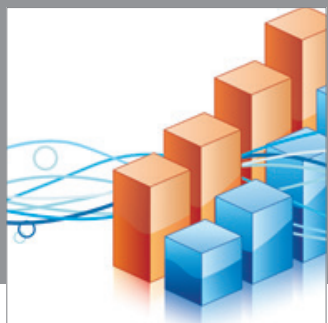

Advances in

Operations Research

mansans

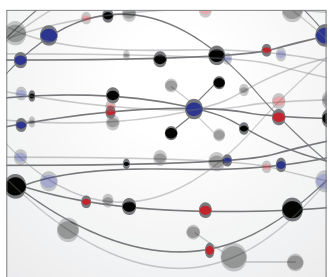

The Scientific World Journal
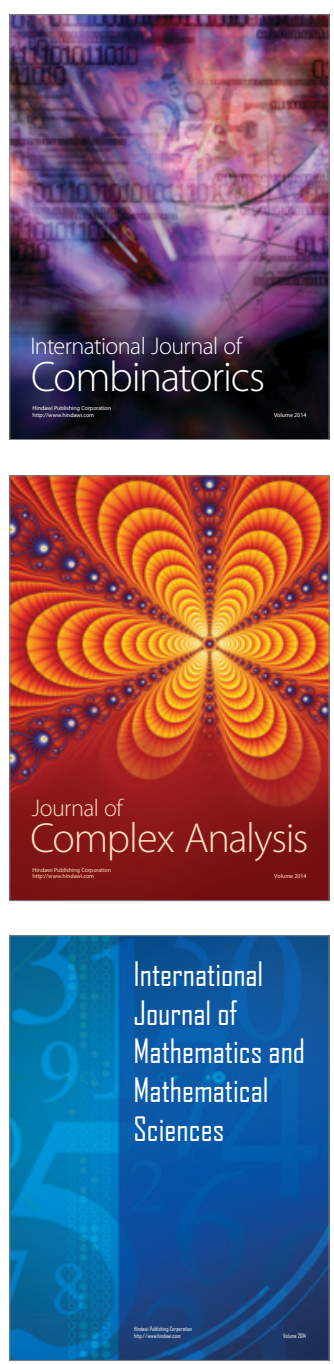
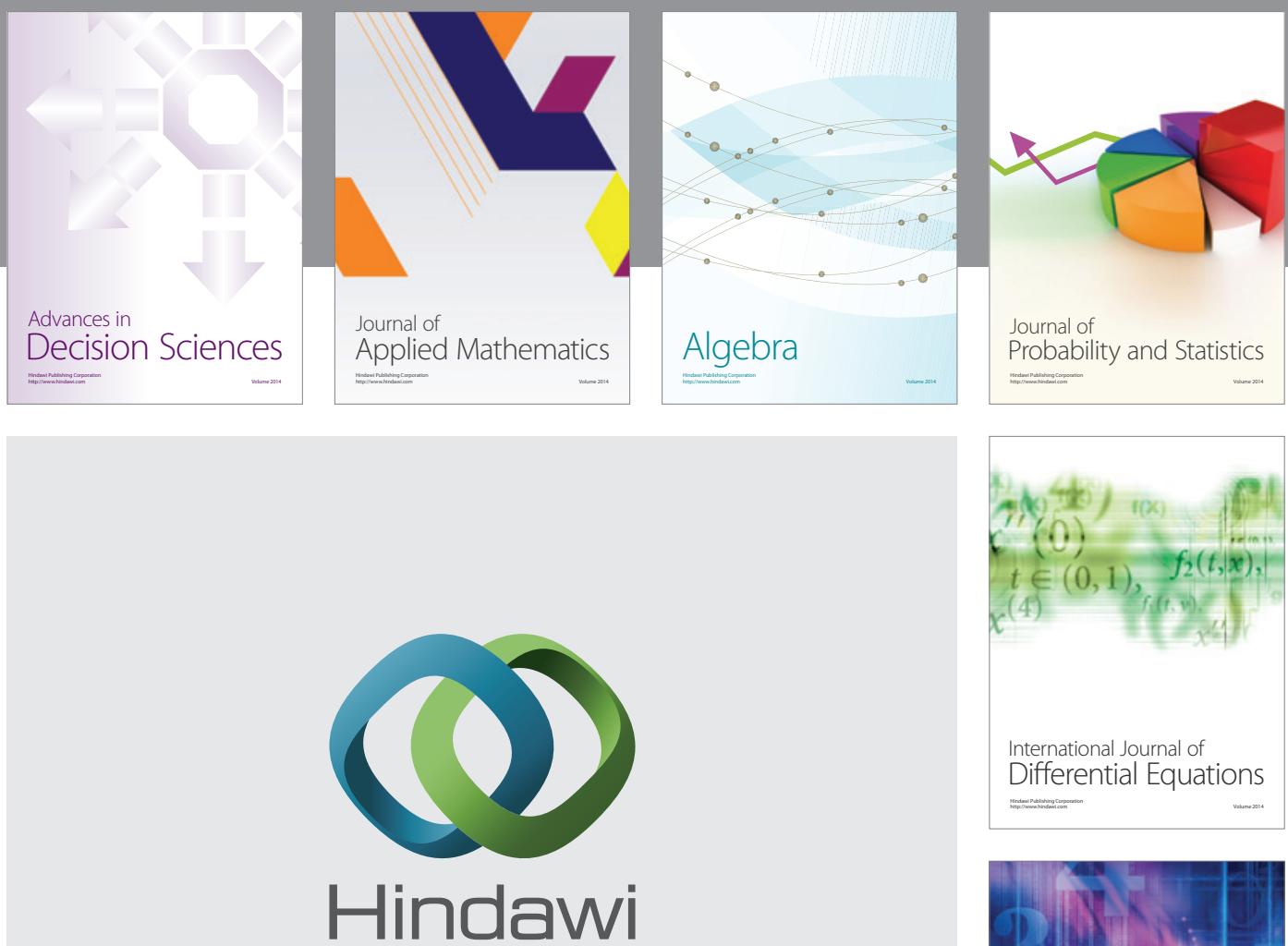

Submit your manuscripts at http://www.hindawi.com
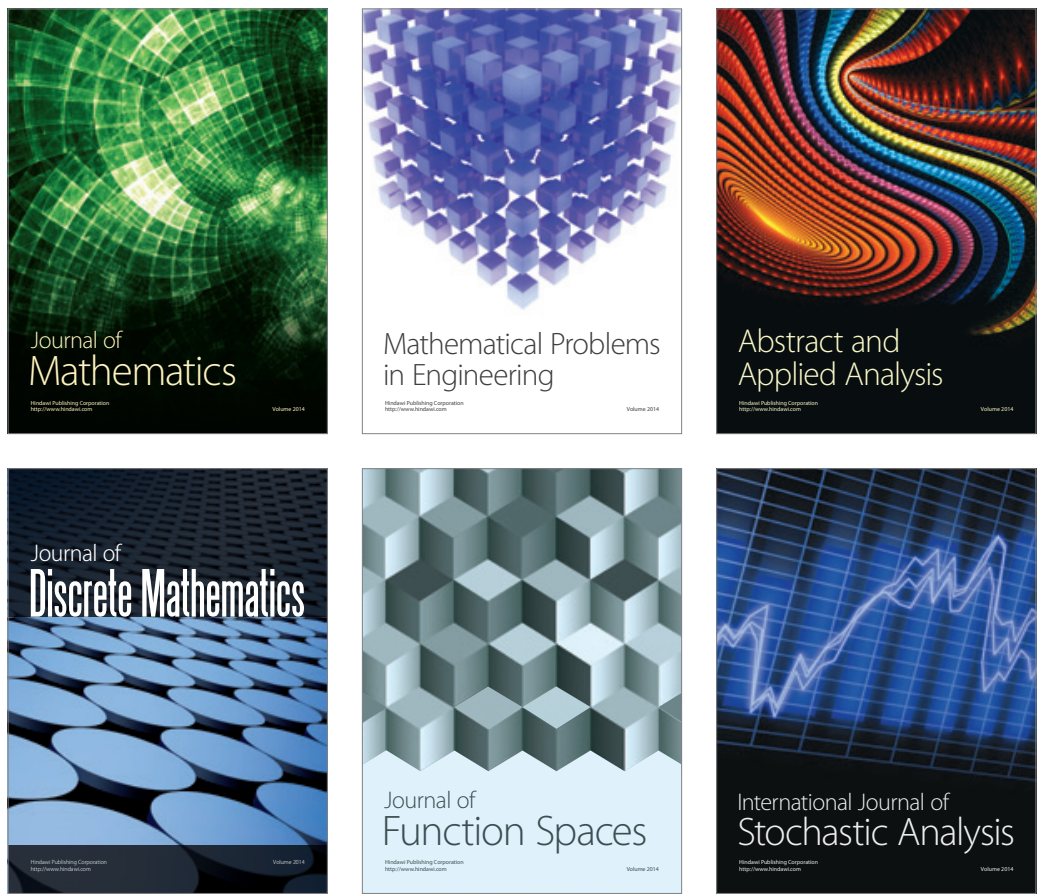

Journal of

Function Spaces

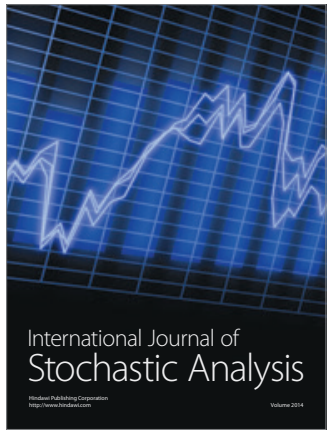

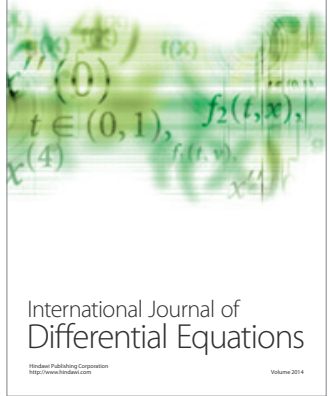
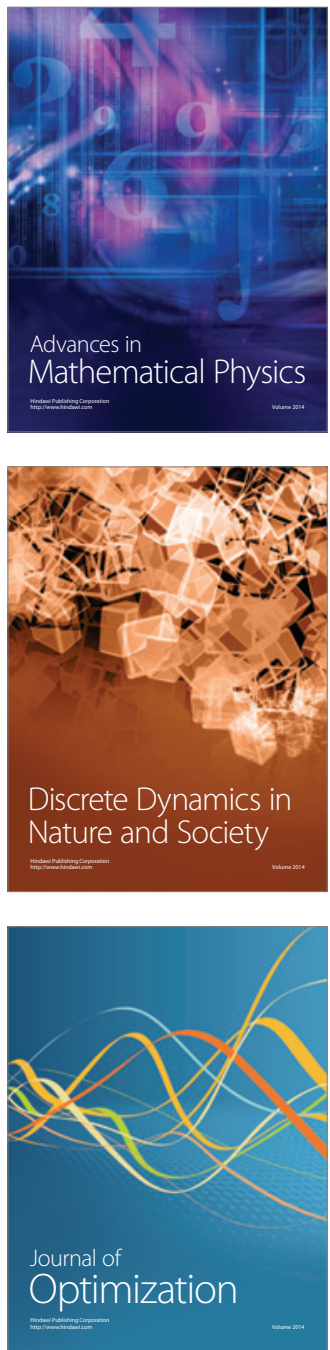